\title{
Gamma/neutron competition above the neutron separation en- ergy in delayed neutron emitters
}

\author{
E. Valencia ${ }^{1, a}$, A. Algora' ${ }^{1,2}$, J.L. Tain ${ }^{1}$, S. Rice ${ }^{3}$, J. Agramunt $^{1}$, A. -A. Zakari- Issoufou ${ }^{4}$, J. Äystö ${ }^{5}$, \\ M. Bowry ${ }^{3}$, V.M. Bui ${ }^{4}$, R. Caballero- Folch ${ }^{6}$, D. Cano-Ott ${ }^{7}$, V. Eloma ${ }^{5}$, T. Eronen ${ }^{5}$, E. Estevez ${ }^{1}$, G.F. \\ Farrelly $^{3}$, M. Fallot ${ }^{4}$, A. Garcia ${ }^{7}$, W. Gelletly ${ }^{3}$, M.B. Gomez-Hornillos ${ }^{6}$, V. Gorlychev ${ }^{6}$, J. Hakala ${ }^{5}$, \\ A. Jokinen ${ }^{5}$, M.D. Jordan ${ }^{1}$, A. Kankainen ${ }^{5}$, F.G. Kondev ${ }^{8}$, T. Martinez ${ }^{7}$, E. Mendoza ${ }^{7}$, F. Molina ${ }^{1}$, \\ I. Moore ${ }^{5}$, A. Perez ${ }^{1}$, Zs. Podolyak ${ }^{3}$, H. Penttilä ${ }^{5}$, A. Porta ${ }^{4}$, P.H. Regan, J. Rissanen ${ }^{5}$, B. Rubio ${ }^{1}$, \\ C. Weber ${ }^{5}$, and IGISOL people \\ ${ }^{1}$ IFIC, CSIC - University of Valencia, E-46071, Valencia, Spain \\ ${ }^{2}$ Institute of Nuclear Research, Debrecen, Hungary \\ ${ }^{3}$ University of Surrey, GU2 7XH, Guildford, UK \\ ${ }^{4}$ Subatech, CNRS/INP2P3, Nantes, EMN, 44307, Nantes, France \\ ${ }^{5}$ IGISOL, University of Jyväskylä, FIN-40014, Jyväskylä, Finland \\ ${ }^{6}$ Universitat Politecnica de Catalunya, 08028, Barcelona, Spain \\ ${ }^{7}$ CIEMAT, 28040, Madrid, Spain \\ ${ }^{8}$ Nuclear Engineering Division, Argonne National Laboratory, IL 60439, Argonne, USA
}

\begin{abstract}
To study the $\beta$-decay properties of some well known delayed neutron emitters an experiment was performed in 2009 at the IGISOL facility (University of Jyväskylä in Finland) using Total Absorption $\gamma$-ray Spectroscopy (TAGS) technique. The aim of these measurements is to obtain the full $\beta$-strength distribution below the neutron separation energy $\left(S_{n}\right)$ and the $\gamma /$ neutron competition above. This information is a key parameter in nuclear technology applications as well as in nuclear astrophysics and nuclear structure. Preliminary results of the analysis show a significant $\gamma$-branching ratio above $S_{n}$.
\end{abstract}

\section{Introduction}

\section{$1.1 \beta$-delayed neutron emission}

In neutron rich nuclei with sufficient neutron excess beta decay can proceed to states above the neutron separation energy in the daughter nucleus, leading to the well-known phenomenon of $\beta$-delayed neutron emission. The neutron width of the resonant states is expected to be much larger than the gamma width, and usually it is assumed that the gamma-ray emission is negligible. This is conventionally the case for estimations of the neutron emission probability based on theoretical calculations of the beta strength distribution [1].

\footnotetext{
a e-mail: ebvalma@ific.uv.es
}

This is an Open Access article distributed under the terms of the Creative Commons Attribution License 2.0, which permits unrestricted use, distribution, and reproduction in any medium, provided the original work is properly cited. 


\section{EPJ Web of Conferences}

\subsection{Total Absorption $\gamma$-ray Spectroscopy (TAGS) technique}

Experimentally the information on $\gamma /$ neutron competition after $\beta$-decay is scarce $[2,3]$. This is due to the fact that the decay populates a region of high excitation energy and high level density and the consequent difficulty to detect the electromagnetic de-excitation with conventional high-resolution spectroscopy using HPGe. $\gamma /$ neutron competition above the $S_{n}$ is readily measured in neutron capture reactions but this information is restricted to nuclei close to the stability for which stable targets can be produced. Far from stability the TAGS technique applied to $\beta$ - decay studies [4-6] offers a unique opportunity to access experimentally this information.

The advantage of this technique lies in its capability of precise determination of the $\beta$-intensity $\left(I_{b}\right)$ - or the related $\beta$-strength $\left(S_{b}\right)$ - distribution as a function of excitation energy in the daughter nucleus. The technique measures the full disintegration $\gamma$-cascades that occur after the $\beta$-decay rather than detecting the individual $\gamma$-rays. In contrast with high resolution (HR) spectroscopy, the TAGS technique overcomes the systematic and common error known as "Pandemonium effect" [8]: from the level scheme obtained in HR, the $\beta$-decay probability is extracted based on the $\gamma$-ray intensity balance; so, for those decays with high fragmentation of the $\gamma$-intensity and primary $\gamma$-rays of high energy - large $Q_{\beta}$ value_, detecting weak $\gamma$-branches or $\gamma$-rays of high energy is difficult because of the limited peak efficiency of Ge detectors. As a consequence, a resulting level scheme is incomplete and excessive $\beta$-intensity is assigned to low-energy excited levels.

\section{Experimental setup}

The total absorption $\gamma$-ray spectrometer used during the experiment was Rocinante (Fig.1(a)): a $4 \pi$ detector composed of $12 \mathrm{BaF}_{2}$ crystals, with a compact size, $\gamma$-ray cascade multiplicity determination and possible neutron discrimination capability. The individual detectors are positioned in a cylindrical geometry, with $25 \mathrm{~cm}$ external diameter, $25 \mathrm{~cm}$ length, and a $5 \mathrm{~cm}$ diameter longitudinal hole. A schematic view of the $12 \mathrm{BaF}_{2}$ crystals, produced with a Monte Carlo (MC) simulation code can be seen in Fig.1(b).

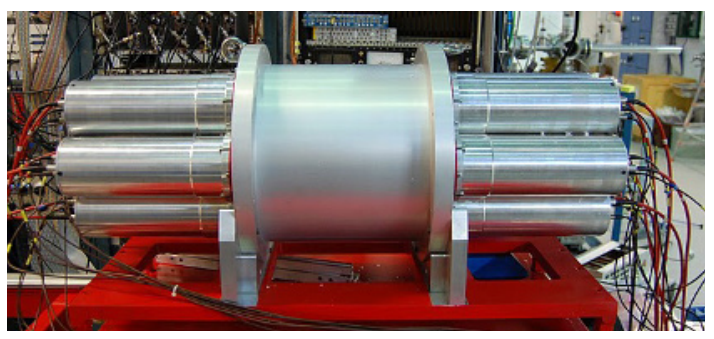

(a) Side view of the total absorption spectrometer.

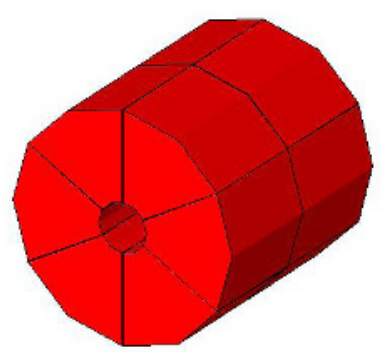

(b) Computer reconstruction of the 12 $\mathrm{BaF}_{2}$ crystals using Geant 4 .

Figure 1. Total absorption $\gamma$-ray spectrometer.

$\mathrm{BaF}_{2}$ has an intrinsic contamination of radium, this is the reason why the background spectra of the spectrometer are dominated by $\alpha$-peaks coming from the decay of ${ }^{226} \mathrm{Ra}$ (which is chemically analogous to $\mathrm{Ba}$ ). One can profit from these impurities, using their peaks as references for the gain stabilization of the individual crystals. The gain stabilization constitutes an essential feature of the electronics and the Data ACQuisition (DACQ) system used. 


\section{Data analysis}

Before data analysis, the raw data must be free of contaminants, such as environmental background, daughter nuclei contribution, summing-pileup effects in the electronic chain as well as the $\gamma$-rays coming from the interaction of $\beta$-delayed neutrons with the detector. Figure 2 shows the calculated contribution of the different components to the total absorption spectrum.

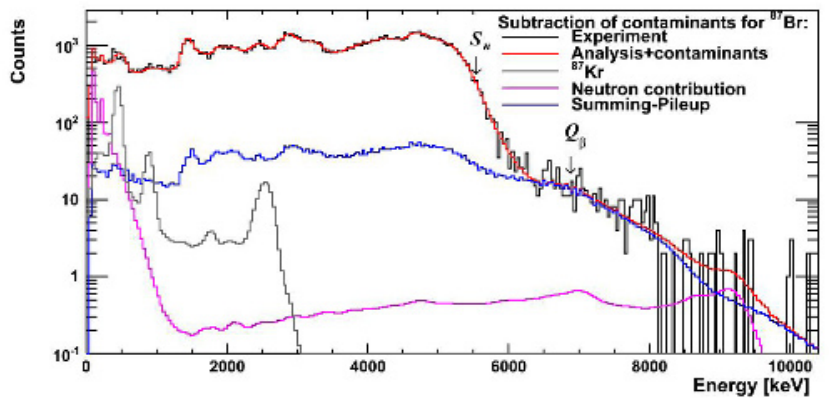

Figure 2. Total absorption spectrum in coincidence with the Si detector of the decay of ${ }^{87} \mathrm{Br}$ plus contaminants.

\subsection{The TAGS inverse problem}

Since a real total absorption spectrometer is not $100 \%$ efficient, the $\beta$-intensity distribution has to be extracted from the measured spectrum by means of a deconvolution procedure. This requires solving the inverse problem:

$$
\mathbf{d}=\mathbf{R}(\mathbf{B}) \cdot \mathbf{f}
$$

where $\mathbf{d}$ represents the measured data, $\mathbf{R}$ is the response matrix of the detector, which depends on the branching ratios $\mathbf{B}$ of the levels in the daughter nucleus and $\mathbf{f}$ is the feeding distribution we wish to determine [7].

To solve it, the branching ratio matrix have been modelled using the known level scheme at low excitation and the statistical nuclear model. Then, using Monte Carlo techniques we calculate the response of the detector to the assumed decay. In this way, it becomes a linear inverse problem and we can apply one of the existing methods of solution, such as the Expectation- Maximization (EM) algorithm in the present case.

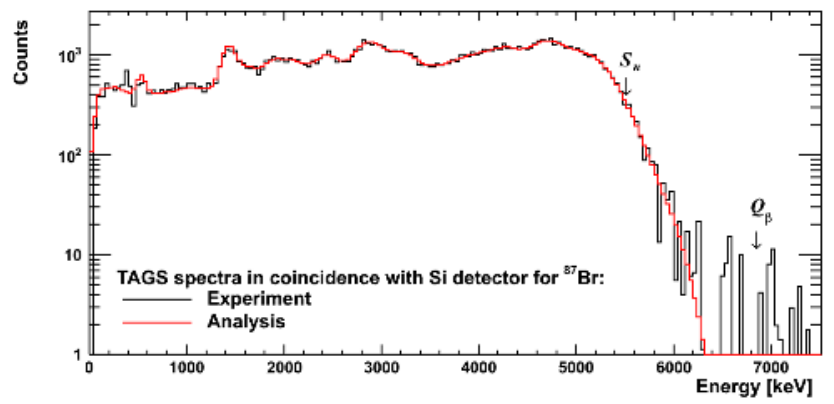

Figure 3. TAGS spectrum in coincidence with the Si detector of the decay of ${ }^{87} \mathrm{Br}$-without contaminantscompared to the spectrum reconstructed from the $\beta$-intensity distribution obtained after solving the inverse problem. 


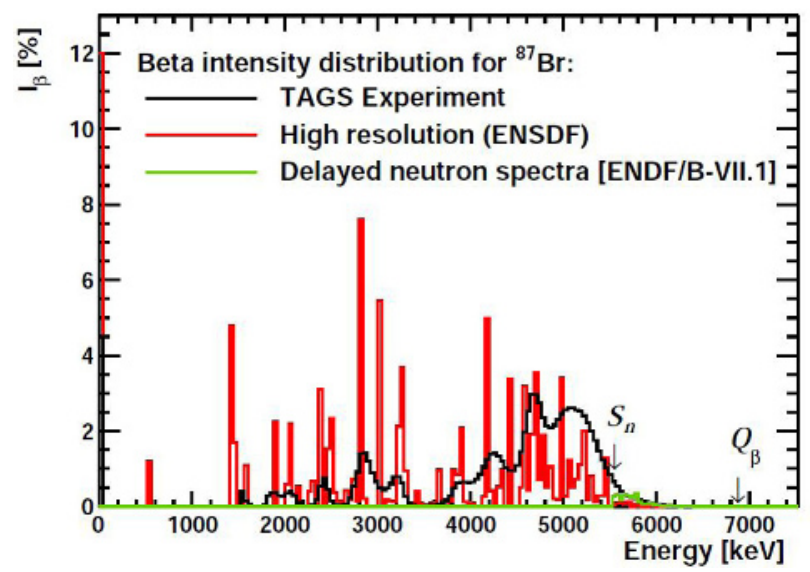

Figure 4. Comparison of the deduced TAGS $\beta$-intensity distribution with earlier high resolution results and the delayed neutron intensity distribution.

\section{Results}

The preliminary results of the analysis of the $\beta$-decay of ${ }^{87} \mathrm{Br}$ using the TAGS technique are shown in Fig. 4 where it can be observed that the fraction of $\beta$-decay intensity above $S_{n}$ going by $\gamma$-ray deexcitation is larger than previously known from HPGe spectroscopy [9-12]. This fraction is comparable to the one proceeding by neutron emission. This surprising result is in contradiction with the general assumption of neutron emission domination above $S_{n}$. We have found a similar trend in other delayed neutron emitters. Currently the reason for this domination is being investigated.

\section{Acknowledgments}

This work has been supported by the Spanish Ministry of Economy and Competitiveness under both projects: FPA2008-06419-C02-01 and FPA2011-24553.

\section{References}

[1] P. Möller, B. Pfeiffer and K.-L. Kratz, Phys. Rev. C 67, 055802 (2003)

[2] D.R. Slaughter, F.M. Nuh, A.A. Shihab-Eldin and S.G. Prussin, Phys. Lett. 38B, 22 (1972)

[3] F.M. Nuh, et al., Phys. Lett. 53B, 435 (1975)

[4] J.L. Tain and D. Cano-Ott, Nucl. Instr. and Meth. A571, 728 (2007)

[5] A. Algora, et al., Phys. Rev. Lett. 105, 202501 (2010)

[6] D. Jordan, et al., Phys. Rev. C 87, 044318 (2013)

[7] D. Cano-Ott, et al., Nucl. Instr. and Meth. A 430, 333-347 (1999)

[8] J.C. Hardy, L.C. Carraz, B. Jonson and P.G. Hansen, Phys. Lett. B 71, 307 (1977)

[9] R. G. Helmer, Nuclear Data Sheets 95, 543 (2002)

[10] S. Raman, et al., Phys. Rev. C 28, 2, 602-622 (1983)

[11] F. M. Nuh, et al., Nucl. Phys. A 293, 410 (1977)

[12] H. Tovedal and B. Fogelberg, Nucl. Phys. A 252, 253 (1975) 